\title{
DISSECTING ANEURYSM OF THE AORTA
}

\author{
By D. A. Pyke, M.B.(Camb.), M.R.C.P. \\ Senior Medical Registrar, Radcliffe Infirmary, Oxford
}

\section{Definition}

Dissecting aneurysm of the aorta is a splitting of the wall of the artery by haemorrhage into the media starting from a tear in the intima or from rupture of vasa vasorum.

\section{Incidence}

Dissecting aneurysm is the cause of about on in a hundred of sudden deaths and about one in four hundred of all hospital deaths. Men are affected much more commonly than women in a proportion of between two and three to one, except in extreme old age when the sex difference is less, partly because there are more old women than old men.

Dissecting aneurysm can occur at any age. The heaviest incidence is in later life, particularly between the ages of 50 and 70 , but one case in five occurs under the age of 40 . This contrasts with an equivalent figure for cardiac infarction under the age of 40 of about one case in fifty.

Of cases occurring in young women, about half are in late pregnancy or soon after delivery. It is uncommon for dissecting aneurysm to develop during labour.

\section{Pathogenesis}

Dissection does not occur in normal arteries. In nearly all cases of dissecting aneurysm there is evidence of degeneration of the media with loss of elastica and muscle resulting in an appearance of cystic degeneration (Fig. I). The media may be so degenerate that at post-mortem an apparently normal part of aorta, separate from the site of dissection, can be split into two coats by gentle rubbing between finger and thumb. The degeneration is much more marked than in the normal loss of smooth muscle with age.

The cause of the cystic necrosis of the media is not known. It has been produced in rats by feeding with the sweet pea Lathyrus odoratus, whose toxic component is $\beta$-amino proprionitrile (Bachhuber et al., 1955), and a medial degeneration has been produced in guinea pigs and rabbits by deprivation of vitamin E (Loewi, r955).

Cystic medial necrosis may be found in patients dying of other conditions. Reid (personal communication) has studied 70 aortas from consecutive post-mortem examinations of patients aged ig to 84 years and has found some medial necrosis in six, but in only one was it severe. There was some association with age but none with sex or hypertension in the six cases. Rottino (1939) found eight cases of advanced medial necrosis in 2 ro consecutive post-mortem examinations, the incidence being greatest in patients who had been hypertensive. He found some medial degeneration in 95 cases.

Medial necrosis is most frequent in the ascending aorta, common in the arch, less so in the descending aorta and only rarely found in any other vessel.

Although medial necrosis appears to be the most important lesion in dissecting aneurysm, high blood pressure is a very common finding. About 90 per cent. of patients suffering from dissecting aneurysm over the age of 40 show evidence of past or present hypertension.

Atheroma plays little and syphilis no part in the pathogenesis of dissecting aneurysm.

Why dissection begins so often in the ascending part of the aorta, why a cystic medial necrosis is almost entirely confined to the aorta and why high blood pressure is so very common a finding are problems that have not yet been solved. To say that the ascending aorta is subject to the greatest mechanical strains, that the strains will be all the more in hypertension and that it is reasonable to expect a diseased artery to give way first at this point is the best answer one can give.

\section{Dissecting Aneurysm in Young Subjects}

The pathogenesis of dissecting aneurysm in patients under 40 differs from that in older patients. High blood pressure is a much less important factor, being present in no more than half. Congenital abnormalities such as coarctation of the aorta, bicuspid aortic valves, septal defects and arachnodactyly, are found in more than half. The association between dissecting aneurysm and coarctation is particularly important. About 30 per cent. of patients under the age of 40 with 


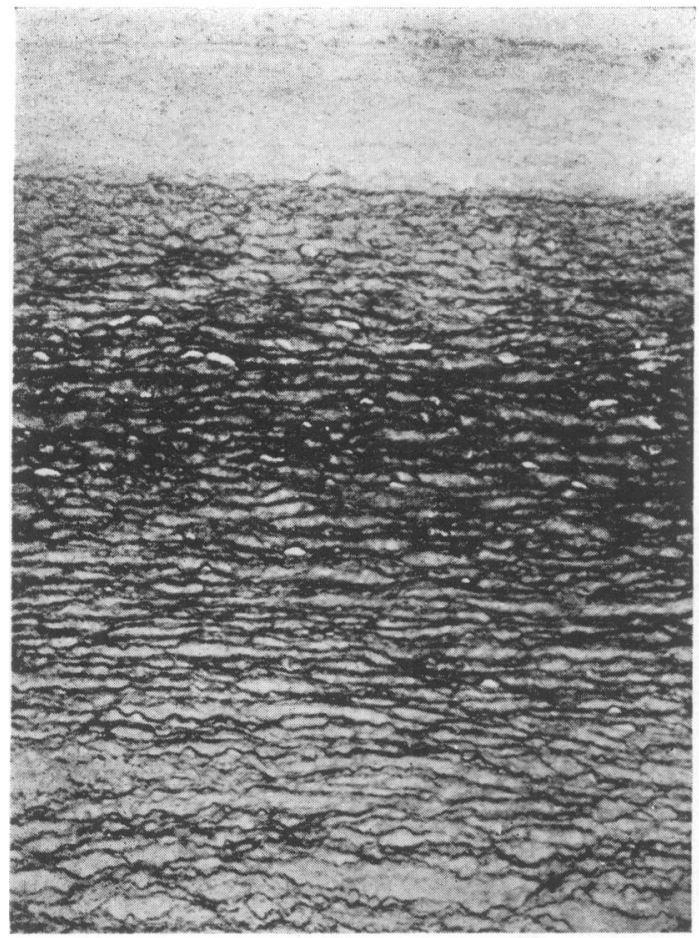

A

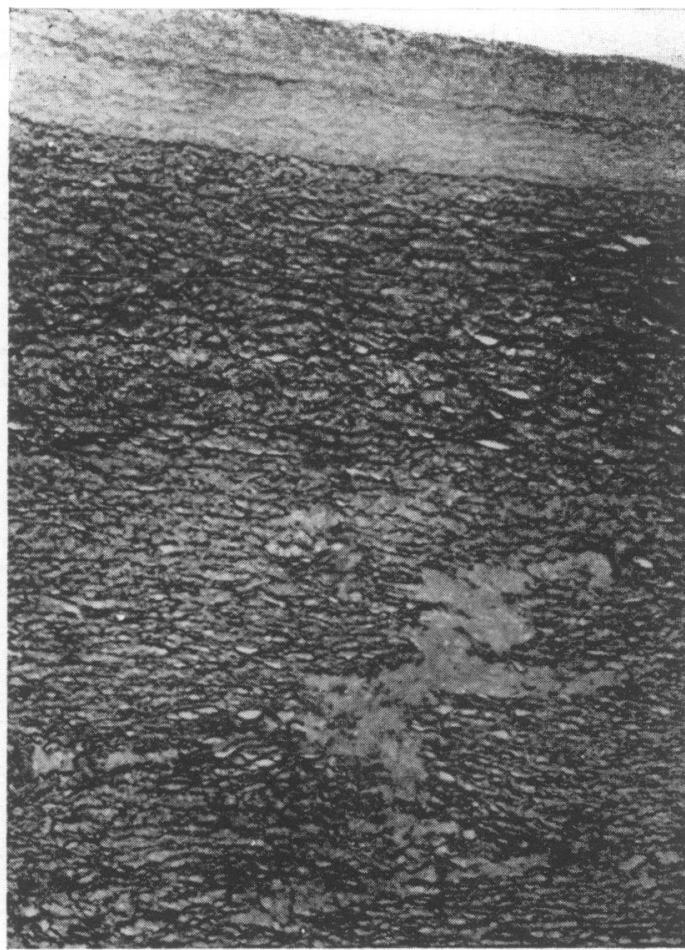

B

FIG. 1.-Sections of aorta $\left(\begin{array}{l}x \\ 6\end{array}\right)$ showing (a) normal structure and (b) necrosis of the media with loss of elastica and cyst formation.

dissecting aneurysm show some degree of coarctation (Schnitker and Bayer, 1944). Thirty-three of Abbott's 200 cases of coarctation died from rupture of the aorta (Abbott, 1928). One-third of the cases of Marfan's syndrome (arachnodactyly, skeletal deformities, high arched palate, abnormalities of the teeth and dislocation of the lens) have congenital abnormalities of the aorta and half of these suffer from dissecting aneurysm (Goyette and Palmer, 1953).

It is probable that there is a congenital disorder of connective tissue in these syndromes, but it is unlikely that medial necrosis develops long before the dissection occurs (Gore, 1953).

\section{Mechanism of Dissection}

The dissection begins as a rupture of an intimal plaque or as haemorrhage from vasa vasorum, usually within a few centimetres of the aortic valves. It spreads rapidly splitting the wall of the aorta into two parts round part or all of its circumference. It may spread proximally to involve the aortic ring, the coronary arteries and the heart muscle and may rupture into the pericardium. Distally it may involve the innominate, carotid, subclavian, intercostal, splanchnic and renal arteries. Since only part of the circumference of the aorta may be involved, the dissection often takes a sinuous course affecting or missing branches capriciously. The dissection affects the larger arteries by spreading along them for a short distance thereby narrowing the lumen. It seldom spreads into the intercostal vessels but tears them from their roots at the aorta. This is presumably due to the difference in angle of attachment and in size of lumen.

Although cases have been described of dissection reaching as far as the popliteals, they are extremely rare and it is usually confined to the aorta and the roots of its branches. Dissection into branch arteries is stopped within a few centimetres of their origin by the normal media.

Dissection may end in one of three ways. Most commonly it ruptures externally into the pericardium, mediastinum, pleura or retro-peritoneal space and this is likely to be fatal, although not necessarily immediately. It may rupture back into the lumen of the aorta leaving a double channel, which is the usual condition in patients who survive. Finally, the dissection may come 
to a stop in the aortic wall and eventually heal by fibrosis.

\section{Clinical Types of Dissecting Aneurysm}

The type of dissecting aneurysm discussed here which occurs at any age, is associated with cystic medial necrosis, starts in the thoracic aorta and spreads for several centimetres in the wall of the aorta is to be distinguished from the rupture of the aorta occurring only in the elderly, associated always with severe atheroma beginning in the abdominal aorta and dissecting for only a short distance if at all.

\section{Clinical Features}

The patient is struck by pain, sometimes gradual and bearable, usually sudden and terrible. The pain is as bad as that of any other disease. Occasionally it is provoked by exertion and may be preceded by a short warning attack.

The distribution of the pain varies with the site of dissection. Usually it is felt in the chest, particularly in the back of the chest, and radiates widely, spreading upwards to the throat and neck and downwards to the abdomen, loins and groins. The pain may start in the lumbar region and spread upwards or forwards into the abdomen. In the majority the pain is as bad or worse in the back as in the front, whether of chest or abdomen. Spread to the arms is not common. Pain of one arm may be due to dissection occluding a subclavian and will then be accompanied by signs of arterial obstruction. Pain of a leg will probably be due to iliac artery occlusion.

Restriction of the pain to the front of the chest is not usual. Of 18 cases of dissecting aneurysm admitted to the Radcliffe Infirmary in the years 1950-I955, the pain was confined to the precordium in only three.

Not only does the pain of dissecting aneurysm tend to be more severe than that of cardiac infarction, but it usually lasts longer. One injection of morphine is seldom enough to relieve it. The pain may last for days and finally disappear only after a week or more.

In a few patients, perhaps one-fifth, there is no pain. The patient may suddenly lose consciousness or become breathless. Haemoptysis, haematemesis or haematuria may occur. Other symptoms are mental confusion, convulsions and weakness of a limb or of one side of the body.

\section{Physical Signs}

There are no pathognomonic signs of dissecting aneurysm. The patient is likely to be much distressed. Vomiting is common. The blood pressure is found to be raised on admission to hospital in 60 per cent. of cases, often to very high levels, and the heart is usually enlarged. The pulse may be abnormal from auricular fibrillation, or partial or complete heart block.

Most of the signs are produced by arterial occlusion from the dissection. Retrograde dissection involving the aortic ring produces an aortic diastolic murmur, often very loud with a thrill, in at least one quarter of the cases. Rarely this leads to a mitral diastolic murmur (Austin Flint). A systolic murmur at base or apex is very common. Most patients will show at least one cardiac murmur. Very occasionally a pericardial rub is heard and even more rarely a pericardial effusion is found.

Dissection of major branches leads, if a carotid is involved, to hemiplegia, if a limb artery is affected to weakness, coolness and slight discolouration of an arm or leg with impaired power, sensation and reflexes. Occasionally a murmur may be heard and a thrill felt over a peripheral artery. Intercostal artery rupture leads to pleural effusion, nearly always left-sided, and less often to paraplegia.

The curious features of the signs of arterial obstruction, particularly of the femoral and spinal arteries, are that they may be transitory-a complete paraplegia may recover within minutes or hours-and that at necropsy no arterial obstruction may be found to account for the physical signs. Thus, a man of 64 was found to have strikingly weaker pulses in the left leg than in the right and a difference in temperature, but at post-mortem examination it was found that the dissection reached only to the coeliac axis.

Involvement of the mesenteric vessels may produce abdominal distension and rigidity and of the renal arteries haematuria. If the dissecting aneurysm has ruptured externally, swellings of the groin or a mass in the abdomen may be felt. There may be faint blue staining of the skin of the neck from extravasated blood.

Proteinuria is extremely common whether the renal arteries are dissected or not.

\section{Diagnosis}

This may be difficult but is often easy. A correct ante-mortem diagnosis was made in 14 out of 18 cases admitted to the Radcliffe Infirmarywith varying degrees of assurance.

Diagnosis depends upon a combination of clinical features, none conclusive but together suggestive: site, severity and distribution of the pain, hypertension, aortic diastolic murmur (the most helpful single sign) peripheral arterial occlusion (particularly affecting carotid or subclavian) and neurological disorders, especially hemiplegia, monoplegia or paraplegia.

Clinical findings may be augmented by in- 


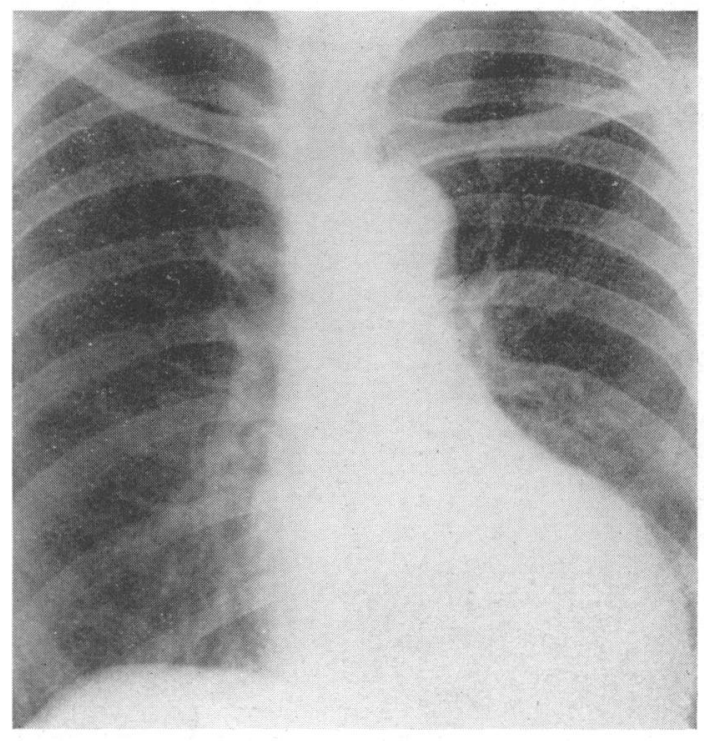

A

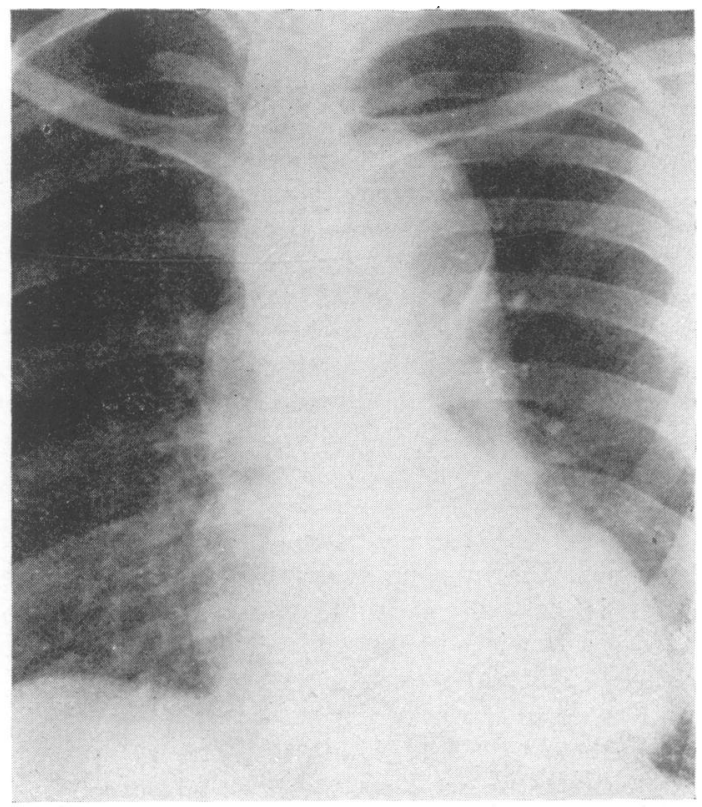

B

FIG. 2.-Chest radiographs (a) two years before and (b) five months after the development of a dissecting aneurysm showing the change in the size of the aorta.

vestigations. The chest $\mathrm{X}$-ray is seldom norma!. Left ventricular hypertrophy and widening of the aortic shadow are common. Signs of pleural or pericardia! effusion may be seen. There may be widening of the superior mediastinal shadow extending over the apices of the lungs due to bleeding into the mediastinum. A dissected innominate or subclavian artery may be seen as a wider shadow than normal. There are two radiological signs which are diagnostic of dissecting aneurysm (and are therefore the only means by which a positive diagnosis can be reached). The first is widening and unfolding of the arch of the aorta compared to previous pictures (Fig. 2). With the increasing practice of chest radiography this has become an occasional help. Thickening of the media of the aorta may be demonstrated if there is intimal calcification in the arch by seeing an unusually wide gap between it and the outer border of the aorta (Fig. 3).

The electrocardiogram is generally abnormal. Evidence of left ventricular hypertrophy is very common. There may be other changes, such as elevation of the S-T segment and inversion of the $T$ wave in some leads but $Q$ waves are se!dom seen and the changes are not those typical of myocardial infarction. Even when the coronary arteries are compressed or occluded, myocardial infarction is very rare. Heart block, complete or incomplete, may develop from damage to the heart muscle by the spreading haematoma. Auricular fibrillation is found in a few cases. The changes of pericarditis, due to blood in the pericardium, are occasionally seen and are of grave import.

Laboratory investigations are of no help in the diagnosis of dissecting aneurysm. An increase of erythrocyte sedimentation rate, to as high as 100 $\mathrm{mm}$. in one hour, will be observed if the patient lives long enough and will disappear in four to eight weeks. Leucocytosis is usual; the average figure in nine cases was 14,500 white blood cells per cubic $\mathrm{mm}$.

\section{Differential Diagnosis}

The conditions which dissecting aneurysm may resemble are several, cardiovascular, abdominal, thoracic and neurological. It may be distinguished from (I) coronary occlusion by the distribution of the pain, the persistence of hypertension and the absence of the characteristic electrocardiographic changes; arterial embolism or thrombosis by the pain in the trunk; subacute bacterial endocarditis with ruptured valve cusp by age, pain, absence of previous illness and high blood pressure; (2) acute abdominal emergencies, such as perforation of the bowel, acute pancreatitis, mesenteric thrombosis, acute cholecystitis and intestinal obstruction, and other sudden episodes 


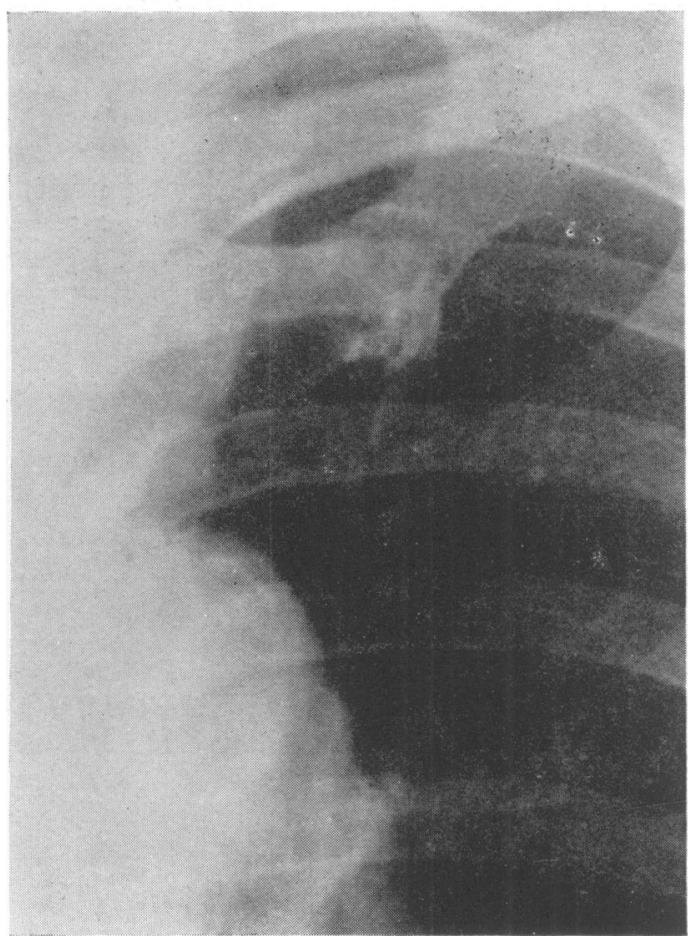

A

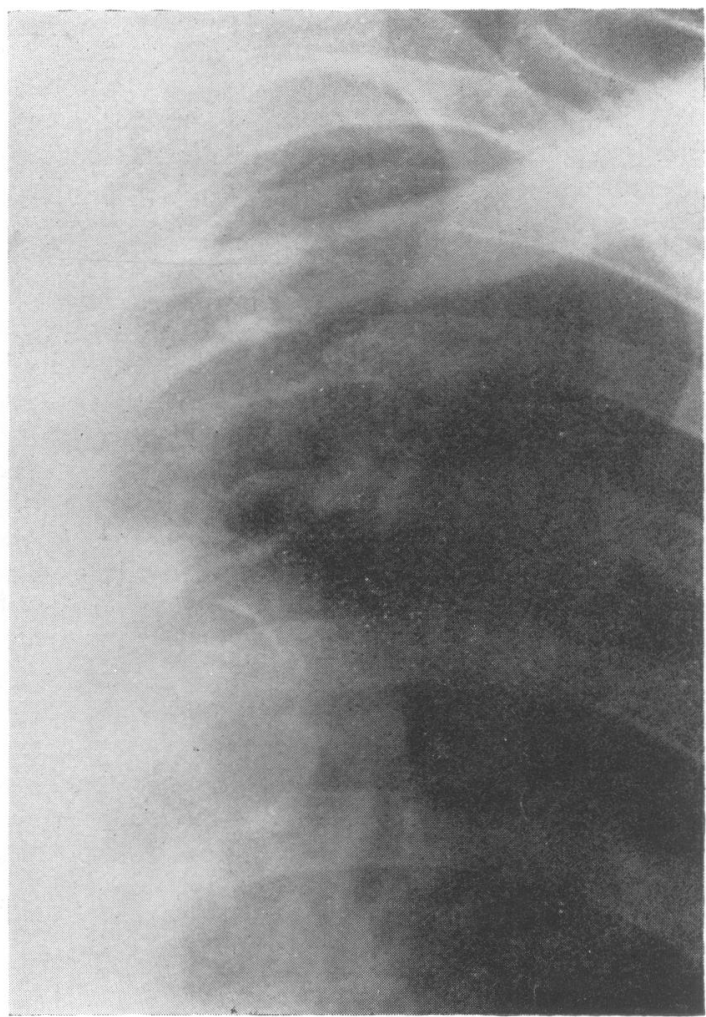

$\mathrm{B}$

FIG. 3.-Chest radiographs (a) four year before and (b) one day after the development of a dissecting aneurysm. The increased thickness of the aortic wall can be seen by comparing the distance between the intimal calcification and the outer border of the aorta.

such as acute peptic ulcer and renal colic, by the positive findings suggesting dissecting aneurysm and the absence of the characteristic signs of these conditions, but even at laparotomy the diagnosis may not be reached (a retro-peritoneal haematoma extending into the groin may look very like an inguinal hernia); (3) cerebrovascular accident, hypertensive encephalopathy, transverse myelitis and spinal subarachnoid haemorrhage by the signs of arterial occlusion, the severe pain and normal cerebrospinal fluid; (4) pulmonary embolism, by the previous good health and probable hypertension; spontaneous pneumothorax by the absence of the characteristic signs.

\section{Course and Prognosis}

Four patients out of five admitted to hospital with dissecting aneurysm die before leaving it, an immediate mortality of 80 per cent. In spite of the fact that the usual cause of death is external rupture, death is usually not instantaneous. The average time of survival of 13 patients who died of dissecting aneurysm in the Radcliffe Infirmary was four days. Four died on the day of admission, six others within a week and three more in the second week after admission. Of the five survivors one died two and one 18 months later of further dissections, and three are alive after four, eight and 36 months.

Frequently a patient appears to improve after the first episode but within a few hours or days deteriorates rapidly from further dissection, usually into the pericardium. Haemopericardium is found in about 80 per cent. of fatal cases. No patient with evidence of pericarditis is likely to survive.

The bigger the dissection the greater the danger but it has to be remembered that clinica! signs of the extent of dissection may be deceptive. An impalpable femoral artery does not necessarily mean that the dissection reaches from the ascending aorta to the femoral canal, although if the pulse remains absent for several hours this becomes probable. Even if the length of a dissection is known it does not mean that all the intervening branches are affected since the dissection may have 
split only part of the circumference of the aorta and have skirted vital branches, such as the renal arteries.

A low blood pressure on admission to hospital is a bad sign. Except in young patients the blood pressure is extremely likely to have been raised before dissection and a fall to normal levels or below usually means a severe dissection with rupture. Of the 18 patients at the Radcliffe Infirmary, five had low or normal blood pressures and all died. Of the remaining 13 , five survived.

If the patient does not die from external rupture of the aneurysm, he is in danger from renal failure. This occurs much more often than dissection of the renal arteries. Mainly because of relative hypotension, renal insufficiency develops and the patient succumbs from uraemia five to ten days after admission. Nearly all patients show some rise of blood urea concentration and proteinuria.

If a patient escapes death from external rupture or renal failure, pain is likely to trouble him severely for the first few days and mildly for longer. His survival will probably be due to the haematoma re-entering the aorta and forming a double vessel but recovery is possible without this by arrest and healing of the dissection. Fever is to be expected and may last one or even two weeks. The temperature may reach $102^{\circ} \mathrm{F}$. Nerve or peripheral vascular signs which last for more than a few hours are likely to persist indefinitely but gangrene of an occluded limb does not develop. Of the patients who survive their illness about one-third die from another dissection, one-third from heart failure and the rest from other causes.

\section{Treatment}

Absolute rest is essential. Pain must be relieved and this needs morphine in adequate doses, repeated if necessary. Later other drugs may be adequate. The patient will need to stay in bed for six to eight weeks.

If the blood pressure remains high it is reasonable to use hypotensive drugs since the risk of rupture is related to the blood pressure. The patient is usually too ill to take drugs by mouth and the need is urgent so ' ansolysen' (pentapyrrolidinium bitartrate) should be given subcutane- ously, starting with a dose of $2.5 \mathrm{mg}$. and repeating and increasing the dose as dictated by hourly recordings of the blood pressure. It is important not to precipitate renal failure by over-treatment. If serious renal failure does develop, it is treated by a high calorie, low protein diet and control of fluid and electrolyte balance, but the outlook is very bad.

Anticoagulants, which might be appropriate treatment for cardiac infarction or arterial occlusion, are to be avoided, although they have been given in error without apparent ill-effect.

Surgeons have not been daunted even by a condition as unpromising as dissecting aneurysm. There are two reports of operations in which an opening was made in the media at the tip of the dissection to allow the blood to re-enter the lumen of the aorta (Gurin et al., 1935; Shaw, 1955). Both patients survived the operation and vascular occlusion of the legs was relieved, but they died about a week later from uraemia.

It is too soon to say whether surgery will have any part to play in the management of dissecting aneurysm. One is tempted to say that it will not, but a few years ago one would have said the same about coarctation of the aorta, aortic stenosis or septal defect. The possibility that it may do should sharpen our appetite for precise diagnosis.

\section{Acknowledgments}

I wish to thank Dr. A. M. Cooke and Dr. A. H. T. Robb-Smith for their help and the consultant staff of the Radcliffe Infirmary for allowing me to consult the notes of patients admitted under their care. I am particularly grateful to Dr. J. V. O. Reid for his very thorough study of the aortas of 70 subjects examined post-mortem.

\section{BIBLIOGRAPHY}

ABВОТТ, М. Е. (1928), Amer. Heart F., 3, 392, 574.

BACHHUBER, T. E., LALICH, J. J., ANGEVINE, D. M., SCHILLING, E. D. and STRONG, F. M. (1955), Proc. Soc. Exp. Biol. and Med., 89, 294.

GORE, I. (1953), Arch. Path., 55, I.

GOYETTE, E. M. and PALMER, P. W. (1 953), Circulation, 7, 373. GURIN, D., EULMER, J. W. and DERBY, R. (1935), New York State F. Med., 35, 1200.

LOEWI, G. (1955), $\mathcal{F}$. Path. Bact. (in the press).

ROTTINO, A. (1939), Arch. Path., 28, 377.

SCHNITKER, M. A. and BAYER, C. A. (1944), Ann. int. Med., 20, 486 .

SHAW, R. S. (1955), New England F. Med., 253, 33 r.

\section{BACK NUMBERS OF THE POSTGRADUATE MEDICAL JOURNAL}

$$
\begin{aligned}
& \text { If any subscribers have copies, in good condition, of JANUARY, FEBRUARY and } \\
& \text { MARCH 1953, the Fellowship of Postgraduate Medicine, 6o Portland Place, London, } \\
& \text { W.I, will be glad to purchase them. }
\end{aligned}
$$

